\title{
Laser-induced incandescence of silicon surface under 1064-nm excitation
}

\author{
A.V. Kopyshinsky, S.E. Zelensky, E.A. Gomon, S.G. Rozouvan, A.S. Kolesnik \\ Taras Shevchenko Kyiv National University, Physics Department \\ 64/13, Volodymyrska str., 01601 Kyiv, Ukraine
}

\begin{abstract}
Laser-induced incandescence (LII) of silicon surface is investigated under the excitation by a Q-switched YAG:Nd laser. With the increase of laser irradiation dose, the increase of LII signal is observed, which is attended by visible changes of the surface geometry. The anomalous behavior of the parameter of non-linearity of LII is observed with the increase of laser excitation power.
\end{abstract}

Keywords: laser-induced incandescence, silicon, laser surface processing.

Manuscript received 27.08.12; revised version received 25.09.12; accepted for publication 17.10.12; published online 12.12.12.

\section{Introduction}

It can be easily illustrated by basic numerical computing that nanosecond-timescale laser radiation with a moderate power density can heat up light-absorbing particles to a few thousand degrees of temperature. As a consequence, thermal radiation of the laser-heated particles within the visible spectral range is observable to the naked eye. This kind of emission under laser excitation is called laser-induced incandescence (LII).

Up to now, LII processes are studied in detail in carbon (soot) nanoparticles that are a product of organic fuel combustion [2-9]. The carbon nanoparticles of a few tens of nanometers in size are present in flames, exhaust gases from engines etc. After exposing to laser irradiation with the energy density of $0.05-0.7 \mathrm{~J} / \mathrm{cm}^{2}$ (power density $10-100 \mathrm{MW} / \mathrm{cm}^{2}$ ), temperature of the nanoparticles increases to a few thousands of Kelvin degrees in a time interval of few nanoseconds. After laser irradiation, the temperature is decreasing slowly with the rate of about $1000 \mathrm{~K} / \mu \mathrm{s}$. Typically, optical signal of LII of soot microparticles is a $0.5-\mu$ s pulse with a nearly black-body radiation spectrum. LII of particulate soot attract interest mostly as a tool for monitoring the amount of microparticles in combustion processes.

LII in the visible spectral interval can be observed under laser irradiation of light-absorbing microparticles suspended in transparent condensed matrices including fluids $[10,11]$, glasses $[12,13]$ and polymers $[14,15]$. Power densities of laser radiation necessary for excitation of detectable LII in these media are 5$50 \mathrm{MW} / \mathrm{cm}^{2}$ for polymers and fluids and 100 $200 \mathrm{MW} / \mathrm{cm}^{2}$ for glass matrices (with $15-20 \mathrm{~ns}$ laser pulse durations). LII in fluids is accompanied by microbubble formation in the vicinity of irradiated microparticles and results in a drastic increase of light scattering. Under the action of a $20 \mathrm{~ns}$ laser pulse, the microbubbles are growing to the sizes comparable with the microparticles' size. As a consequence, self-induced laser pulse attenuation is observed after propagation through the suspension. It is this phenomenon, called optical limiting, that makes light-absorbing suspensions promising for usage as protective elements for photodetectors, devices, eyesight parts etc. against undesirable excessive power levels of pulsed laser radiation.

As to LII of microparticles in solid matrices (solid polymers, glass), such objects have been studied up to date more poorly than aerosols and liquid suspensions.

LII was also observed in silicon nanoparticles (silicon nanopowder) and in porous silicon [16].

New interesting situation arises when laser radiation heats up the absorbing medium skin layers to incandescent temperatures. It is supposed that this kind of LII can provide useful information for laser surface 
treatment, monitoring of surface temperature, roughness, presence of protective layers on the surface etc. As far as we know, these problems haven't been examined yet.

Properties of LII of surface layers haven't been studied enough. For example, paper [17] informs about carbon surface LII under Q-switched YAG:Nd laser irradiation. The obtained data confirm the abovementioned assumption that the surface roughness can significantly influence the parameters of LII.

In this paper, we studied the LII of a crystalline silicon surface under YAG:Nd laser irradiation. The type of studied samples was chosen with account of a few reasons. An important factor of our interest to the processes in silicon was their domination in microelectronics, which results in indefatigable interest. Therefore, the silicon optical properties (also after powerful laser irradiation) are mostly known. Using various laser wavelengths, we could change the heatingup depth of the studied crystal. In this paper, we mostly studied near IR irradiation of silicon surface. The lasing wavelength in this case corresponds to the fundamental absorption edge, which results in low absorption and in the possibility to heat up a relatively thick surface layer of the sample. Also, due to a strong temperature dependence of absorption coefficients of silicon [18, 19], there is a possibility to realize a nonlinear absorption in LII experiments.

\section{Experimental}

In this work, we studied silicon samples made of a $\mathrm{p}$ type boron-doped silicon wafer (SEMI M1-00, Prolog Semicond, Ltd.) of (111) orientation, grown by the Czochralski process, with the resistivity of $0.0001 \mathrm{Ohm} \cdot \mathrm{cm}$.

The setup contained a $\mathrm{Nd}^{3+}$ :YAG pulsed laser with one-pass optical amplifier. The laser operated in a Qswitched regime (wavelength $1064 \mathrm{~nm}$, laser pulse duration $20 \mathrm{~ns}$ ). The laser light was focused on the sample surface by an objective lens with the focal distance of $210 \mathrm{~mm}$ in order to reach the required power densities $\left(100-200 \mathrm{MW} / \mathrm{cm}^{2}\right)$. In this paper, we introduce the light intensity $F_{0}$ averaged over the time interval and within the laser beam cross-section. In the course of our measurements, we performed lasing power fine tuning by changing the pumping energy of the optical amplifier. The sample was irradiated by a sequence of laser pulses with a low repetition rate $(0.5$ 1 pulses per second). Photodetectors registered the energy of each pulse from the sequence. The optical scheme of the LII signal registration included a single grating monochromator tuned to the wavelength $500 \mathrm{~nm}$.

\section{Results and discussion}

We found the incandescence of silicon surface in the visible spectral range to appear above the threshold levels of laser intensity - approximately $150 \mathrm{MW} / \mathrm{cm}^{2}$. The energy of the emitted light was unstable, with significant variations from pulse to pulse. The appearance of LII was accompanied by appreciable changes in the sample surface morphology, which put significant obstacles in our experiments.

The dependences of LII intensity $I_{\mathrm{LII}}$ on the dose of laser irradiation for three different values of laser intensity are presented in Fig. 1. As the dose, we mean here the number of laser pulses $N$ with a fixed intensity $F_{0}$ that irradiated the studied surface. The data presented in Fig. 1 was obtained with one pulse per second repetition rate. Each curve in Fig. 1 was taken in a separate surface spot.

As we can see from Fig. 1 , at $F_{0}=187 \mathrm{MW} / \mathrm{cm}^{2}$ LII appears after preliminary surface irradiation by a few tens of laser pulses, though at $F_{0}=179 \mathrm{MW} / \mathrm{cm}^{2}$ LII does not appear even if $N=100$. The values of $F_{0}$ given in Fig. 1 were obtained after averaging. In the experiments with a sequence of laser pulses, the standard deviation of laser pulse energy was approximately $3 \%$.

It is important to note that the data of $I_{\mathrm{LII}}(N)$ measured with the same $F_{0}$ but in different surface spots slightly differ between each another. The results in Fig. 1 were averaged over a few surface spots.

The appearance of LII after laser irradiation was accompanied by noticeable changes in physical characteristics of the irradiated spots. After irradiation with the values of laser intensity and dose sufficient for appearance of LII, the irradiated surface profile changed significantly.

Atomic force microscopy (AFM) 2D scans and scans cross-sections for different irradiation doses at $F_{0}=181 \mathrm{MW} / \mathrm{cm}^{2}$ are presented in Fig. 2. A typical sample surface scan prior to laser irradiation is presented in Fig. 2a.

Fig. 2 b corresponds to the irradiation dose $N<20$ with no accompanied LII observed. Here we can see rough spots on the sample surface with the height approximately $0.05 \mu \mathrm{m}$.

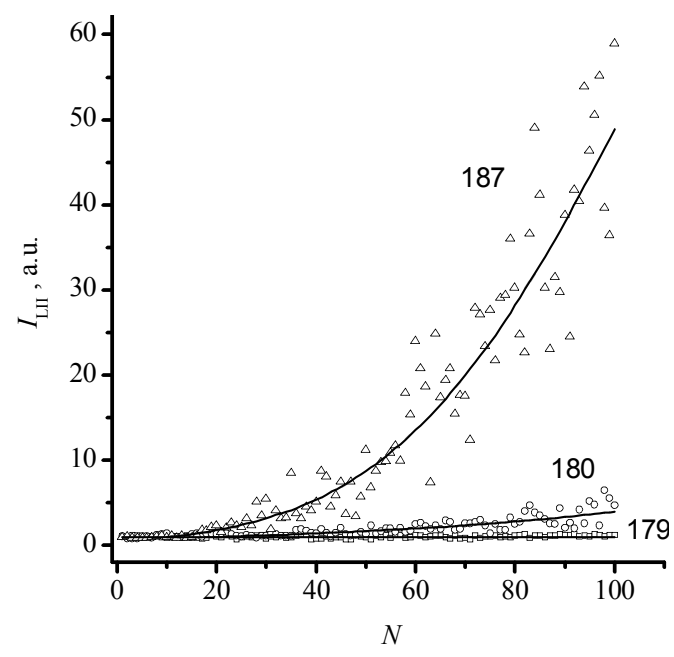

Fig. 1. Silicon surface LII intensity as a function of irradiation dose, where $N$ is the number of laser pulses. Numbers near the curves indicate the laser surface power density in $\mathrm{MW} \cdot \mathrm{cm}^{-2}$. 

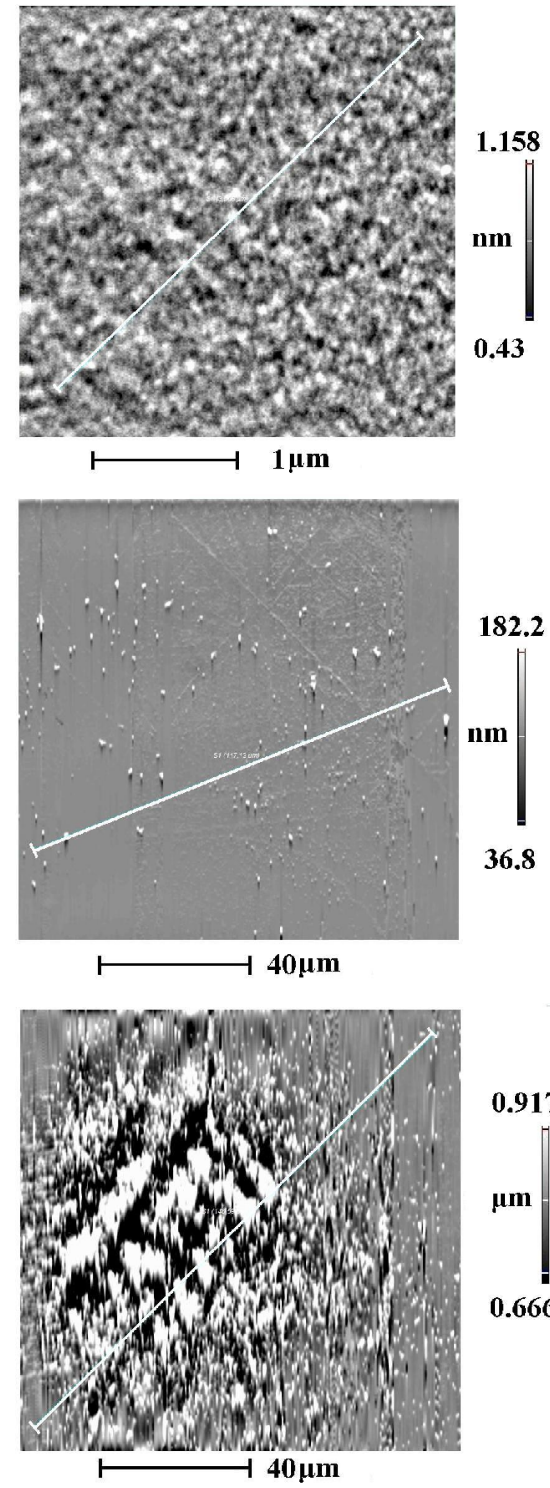

0.917
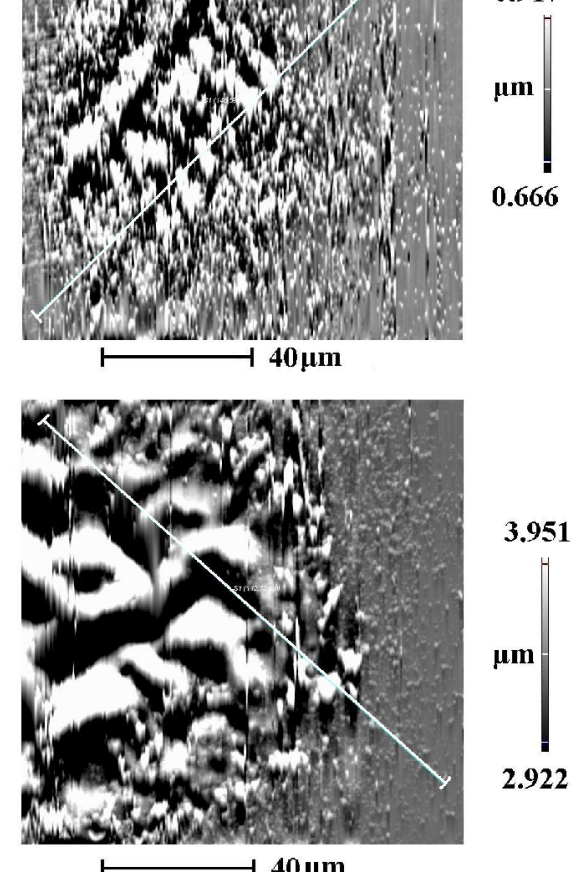

$40 \mu \mathrm{m}$ a)
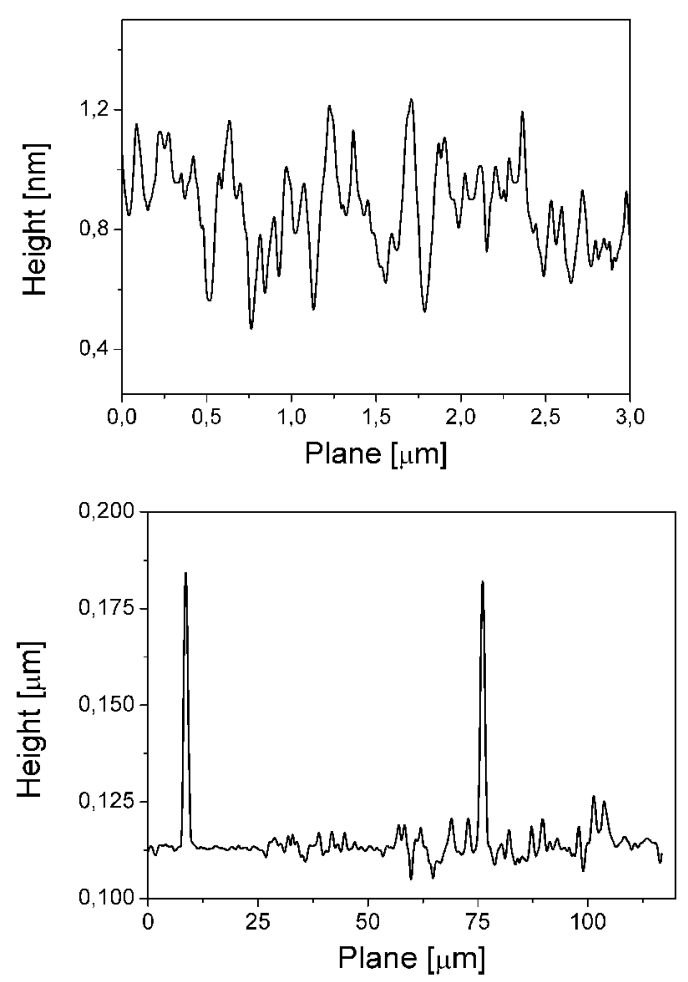

b)

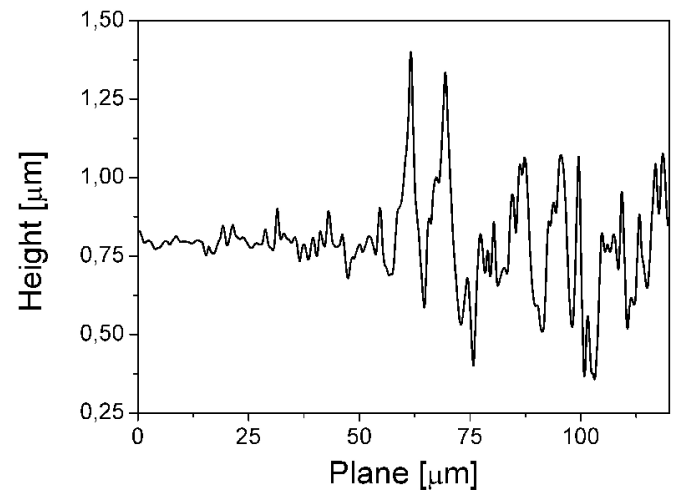

c)

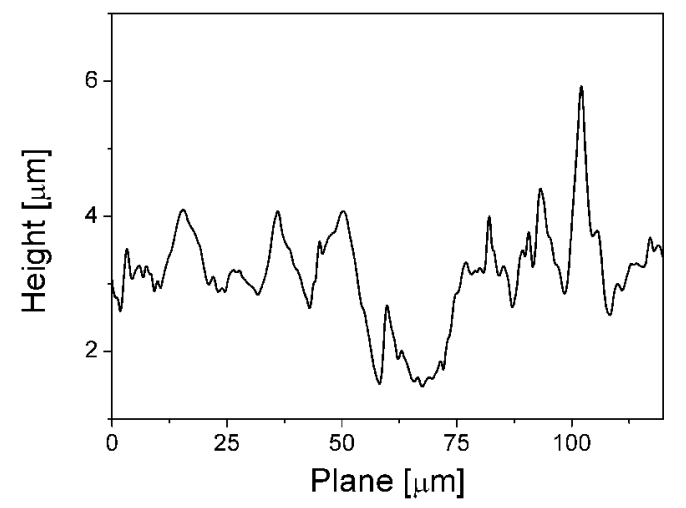

Fig. 2. AFM scans of the silicon surface: (a) non-irradiated region; (b) irradiation dose $N<20$; (c) irradiation dose $N=40$; (d) irradiation dose $N=80$.

Fig. 2c corresponds to the irradiation dose $N=40$. According to Fig. 1, this dose provides increase in the LII intensity with $N$. Thus, as we can see from Fig. 2c, after laser irradiation the surface obtains roughness with typical sizes 5 to $10 \mu \mathrm{m}$ and heights approximately $1 \mu \mathrm{m}$.
With increasing the irradiation dose up to $N=80$, as we can see from Fig. 2d, laser-induced roughness on the sample surface increases up to $10 \ldots 20 \mu \mathrm{m}$ in size and up to $5 \mu \mathrm{m}$ in height. The corresponding LII has a high detectable intensity. 
The above presented specifics of LII buildup following the increase of laser irradiation dose are similar to the results of [20], where scattered laser light intensity from the doped silicon surface was found to become higher after irradiation by a sequence of laser pulses. The laser used in [20] was a $\mathrm{Nd}^{3+}$ :YAG laser with $300 \mathrm{~ns}$ pulse duration and $2-3 \mathrm{MW} / \mathrm{cm}^{2}$ power density.

The above-mentioned dependence of LII intensity on the laser irradiation dose creates some obstacles for further incandescence studies. However, the following moment attracts attention. At $F_{0} \geq 181 \mathrm{MW} / \mathrm{cm}^{2}$ and $N \geq 200$ LII intensity becomes practically independent of a further irradiation dose (at least for a few tens of laser pulses). This behavior provides a possibility to study $I_{\text {LII }}\left(F_{0}\right)$ dependences. Relevant measurements have been performed in a narrow interval of laser power densities below $181 \mathrm{MW} / \mathrm{cm}^{2}$ and $N>200$. At a fixed value of $F_{0} \approx 181 \mathrm{MW} / \mathrm{cm}^{2}$, LII signal was checked to be of the same value before and after the measurements. In Fig. 3, $I_{\text {LII }}\left(F_{0}\right)$ dependences are presented in the double logarithmic scale.

As we can see from Fig. 3, LII intensity is nonlinearly dependent on the laser excitation power. In order to evaluate the nonlinearity, we can introduce similarly to [10] a dimensionless parameter:

$\gamma=\frac{\mathrm{d}\left(\ln I_{\text {LII }}\right)}{\mathrm{d}\left(\ln F_{0}\right)}=\frac{\mathrm{d} I_{\text {LII }} / I_{\text {LII }}}{\mathrm{d} F_{0} / F_{0}}$

Apparently, $\gamma$ characterizes the $I_{\mathrm{LII}}\left(F_{0}\right)$ curve slope in the double logarithmic scale. Typical numbers of the parameter $\gamma$ in glasses and suspensions are 2 to 6 $[10,12]$, for carbon surface approximately 8 [17]. As seen from Fig. 3, $\gamma$ for preliminary irradiated silicon surface is approximately equal to 12 (at LII registration wavelength $500 \mathrm{~nm}$ ).

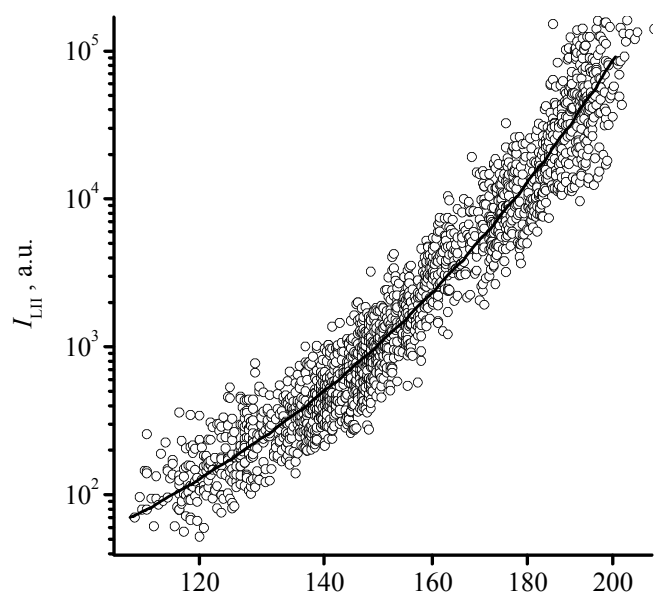

Fig. 3. LII intensity of the irradiated silicon surface as a function of laser excitation intensity (in a log-log scale).
As it was found out in $[10,12]$, the parameter $\gamma$ for suspensions of light-absorbing microparticles depends on the laser excitation power. Usually, $\gamma$ significantly decreases if $F_{0}$ grows. This behavior is typical for LII of absorbing particles because of Planck's law for blackbody radiation. For LII of carbon surface, similar tendency of decreasing $\gamma$ with increasing $F_{0}$ is observed [17]. However, as seen from Fig. 3, this tendency is broken for preliminarily-irradiated silicon surface. At low values of $F_{0}$ in Fig. 3, $\gamma$ is nearby 7, whereas $\gamma$ reaches 16 at high values of $F_{0}$. The corresponding data for $\gamma$ are given in Fig. 4.

Abnormal $\gamma\left(F_{0}\right)$ behavior requires more detailed attention. As we know from $[18,19]$, absorption coefficient for pure silicon at the fundamental absorption band edge (at the wavelength $1064 \mathrm{~nm}$ ) depends drastically on temperature. The coefficient alters from 14 to $2300 \mathrm{~cm}^{-1}$ with the sample heating from 300 up to $1000 \mathrm{~K}$. Apparently, such substantial temperature dependence of the silicon absorption coefficient $\alpha(T)$ causes the anomalous $\gamma\left(F_{0}\right)$ behavior. In favor of this assumption, we can provide the following qualitative explanations. In LII experiments, higher laser intensities provide higher maximal values of temperature. For the irradiated object, the more it gets warm, the more its absorption coefficient $\alpha$ increases, that in turn provides even more increase of the temperature. Thus, we can expect, that in the case of $\frac{\mathrm{d} \alpha}{\mathrm{d} T}>0$ LII signal will grow with $F_{0}$ more quickly than in the case of $\alpha=$ const.

In addition to $\alpha(T)$, the thermal capacity and heat conductivity of silicon also change with temperature [21], which can have an effect on $\gamma\left(F_{0}\right)$ dependence. However, the analysis of how these parameters can influence LII requires numerical computing with the equations of heat conductivity and radiation transport which is not part of this paper.

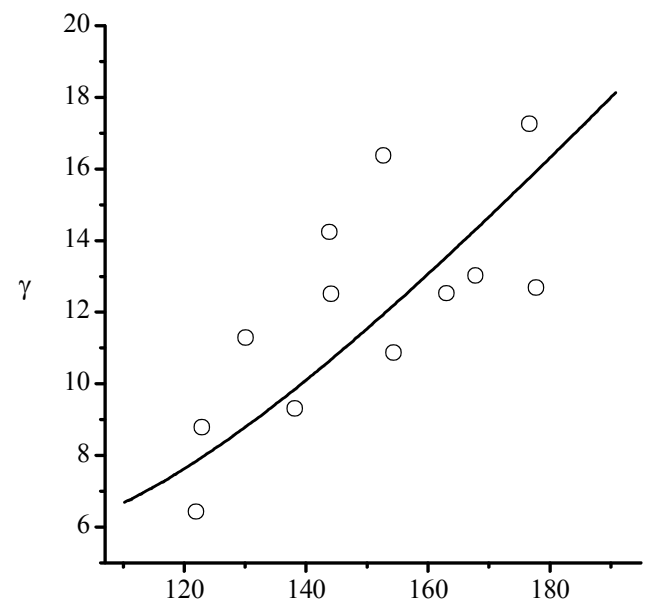

Fig. 4. $\gamma$-parameter for the irradiated silicon surface as a function of laser excitation intensity 
One also has to pay attention to some specifics of laser heating of materials with sharp temperature dependence of the absorption coefficient. We could expect the sample maximal temperature under pulsed laser irradiation will depend significantly on the initial conditions, including the initial values of the absorption coefficient and the initial temperature. Therefore, the absorption coefficient inhomogeneity within the irradiated spot of the surface can result in significant non-uniformity of surface heating even if the laser has homogenous cross-beam power distribution.

For crystalline silicon and germanium under laser irradiation, it is known [22] that surface layers do not melt homogeneously, hence in some time interval the near-surface layer consists of a mixture of solid and liquid phases. The above presented data of laser induced surface shape alterations (Figs. 2b, 2c, 2d) testify that the irradiated spot temperature in our experiments exceeds silicon melting point which equals $1686 \mathrm{~K}$ [21, 23]. Apparently, the after-irradiation depressions and asperities on the silicon surface are formed due to the local melting (or boiling) in the focused spot.

Let us return to LII buildup dynamics after accumulation of the irradiation dose, presented in Fig. 1. The observed behavior of $I_{\mathrm{LII}}(N)$ can be explained by taking into account a few factors.

First, it is plausible to suggest that part of the lasermelted silicon in the surface layer after irradiation becomes amorphous. As the characteristics of amorphous and crystalline silicon differ [23], laserinduced partial or complete amorphisation of the surface layer can significantly influence heating of this surface spot by subsequent laser pulses. In particular, it was shown [21, 24-26] that absorption coefficient of amorphous silicon is much higher than of crystalline silicon. Therefore, laser irradiation of the crystalline silicon surface by a sequence of laser pulses results in gradual accumulation of amorphous phase, which facilitates LII excitation by the subsequent laser pulses. Thus, we can expect further LII buildup in the process of irradiation dose accumulation, as it is observed in the experiments (see Fig. 1).

The second factor explaining the dependence $I_{\text {LII }}(N)$ presented in Fig. 1 can be general warming up of the sample after laser irradiation. Taking into account sample dimensions (surface area approximately $1 \mathrm{~cm}^{2}$, thickness $0.5 \mathrm{~mm}$ ) simple assessments show, each laser pulse from the sequence can heat up the entire sample approximately to $0.1 \mathrm{~K}$. As a result, in the process of laser irradiation by a sequence of pulses, average sample temperature increases slowly with $N$, which results in the increase of efficiency of LII excitation due to the increase of initial absorption coefficient for the subsequent laser pulses.

Finally, the third reason which could result in gradual increase of initial absorption coefficient of silicon in the process of dose accumulation is formation of crystal lattice dislocations. It is known, periodic action of short laser pulses $\left(\tau \sim 10^{-7} \mathrm{~s}\right)$ on the silicon surface results in origination, growth, and self-ordering of lattice defects, and finally the silicon surface destruction is observed [20]. During laser irradiation, energy of formation of point defects in silicon goes down by a few times, hence the number density of defects significantly increases $\left(10^{19}-10^{21} \mathrm{~cm}^{-3}\right)$. High concentration of non-equilibrium point defects results in its fluctuative congestion, e.g. segregation of vacancies, formation of pores and dislocation loops. Further growth of dislocations occurs due to the drain of point defects. The drain of point defects towards dislocations is most effective in silicon at high temperatures, which is typical for laser irradiation process. The dislocations sizes at high irradiation doses depend on the irradiation mode and fluctuate from 10 to $70 \mu \mathrm{m}$ [20].

\section{Concluding remarks}

Summing up, it is necessary to note that in this work we considered the properties of LII of silicon surface under excitation by laser pulses with the photon energy corresponding to fundamental band edge $\hbar \omega \approx E_{g}$. Taking another laser excitation wavelength, e.g. in $\hbar \omega>E_{g}$ spectral range, we can essentially facilitate excitation of LII of silicon surface. However, in this case the absorption coefficient temperature dependence will be weaker, which could result in loss of some special features of LII.

\section{References}

1. C. Schulz, B.F. Kock, M. Hofmann, H. Michelsen, S. Will, B. Bougie, R. Suntz, G. Smallwood, Laserinduced incandescence: Recent trends and current questions // Appl. Phys. B, 83(3), p. 333-354 (2006).

2. L.A. Melton, Soot diagnostics based on laser heating // Appl. Opt., 23(13), p. 2201-2208 (1984).

3. R.L. Vander Wal, K.J. Weiland, Laser-induced incandescence: development and characterization towards a measurement of soot-volume fraction // Appl. Phys. B, 59, p. 445-452 (1994).

4. R.L. Vander Wal, Z. Zhou, M.Y. Choi, Laserinduced incandescence calibration via gravimetric sampling // Combustion and Flame, 105, p. 462470 (1996).

5. C.R. Shaddix, K.C. Smyth, Laser-induced incandescence measurements of soot production in steady and flickering methane, propane, and ethylene diffusion flames // Combustion and Flame, 107, p. 418-452 (1996).

6. M. Hofmann, W.G. Bessler, C. Schulz, H. Jander, Laser-induced incandescence for soot diagnostics at high pressures // Appl. Opt., 42(12), p. 20522062 (2003).

7. K.A. Thomson, K.P.Geigle, M. Kohler, G.J. Smallwood, D.R. Snelling, Optical properties of pulsed laser heated soot // Appl. Phys. B, 104, p. 307-319 (2011). 
8. S. De Iuliis, F. Cignoli, S. Maffi, G. Zizak, Influence of the cumulative effects of multiple laser pulses on laser-induced incandescence signals from soot // Appl. Phys. B, 104, p. 321-330 (2011).

9. H.A. Michelsen, F. Liu, B.F. Kock, H. Bladh, A. Boiarciuc et al., Modeling laser-induced incandescence of soot: a summary and comparison of LII models // Appl. Phys. B, 87, p. 503-521 (2007).

10. S. Zelensky, Laser-induced heat radiation of suspended particles: a method for temperature estimation // J. Opt. A: Pure Appl. Opt., 1, p. 454458 (1999).

11. Ju.Ju. Rulik, N.M. Mikhailenko, S.E. Zelensky, A.S. Kolesnik, Laser-induced incandescence in aqueous carbon black suspensions: the role of particle vaporization // Semiconductor Physics, Quantum Electronics \& Optoelectronics, 10(2), p. 6-10 (2007).

12. S. Zelensky, Laser-induced heat radiation in borate glass // J. Phys.: Condens. Matter, 10, p. $7267-$ 7272 (1998).

13. A.V. Kopyshinsky, Ya.P. Lazorenko, S.E. Zelensky, Laser-induced incandescence of borate glass doped with carbon microparticles // Functional Materials, 18(1), p. 116-120 (2011).

14. S.E. Zelensky, O.V. Kopyshinsky, V.V. Garashchenko, A.S. Kolesnik, V.M. Stadnytskyi, K.S. Zelenska, Ye.V. Shynkarenko, Optical transmittance of carbon suspensions in polymer matrixes under powerful pulsed laser irradiation // Semiconductor Physics, Quantum Electronics \& Optoelectronics, 13(1), p. 70-73 (2010).

15. S.E. Zelensky, A.S. Kolesnik, O.V. Kopyshinsky, V.V. Garashchenko, K.S. Zelenska, V.M. Stadnytskyi, E.V. Shynkarenko, Thermal emission of carbon microparticles in polymer matrices under pulsed laser excitation // Ukr.J. Phys., 54(10), p. 983-988 (2009).
16. P. Roura, J. Costa, Radiative thermal emission from silicon nanoparticles: a reversed story from quantum to classical theory // European J. Phys., 23, p. 191-203 (2002).

17. S.E. Zelensky, L.V. Poperenko, A.V. Kopyshinsky, and K.S. Zelenska, Nonlinear characteristics of laser-induced incandescence of rough carbon surfaces // Proc. SPIE, 8434, 84341H (2012).

18. E.H. Sin, C.K. Ong, and H.S. Tan, Temperature dependence of interband optical absorption of silicon at 1152, 1064, 750, and $694 \mathrm{~nm} \mathrm{//} \mathrm{Phys.}$ status solidi (a), 85, p. 199-204 (1984).

19. C.K. Ong, H.S. Tan, and E.H. Sin, Calculations of melting threshold energies of crystalline and amorphous materials due to pulsed-laser irradiation // Mater. Sci. Eng., 79, p. 79-85 (1986).

20. A.F. Banishev, V.S. Golubev, A.Yu. Kremnev, Generation and accumulation of dislocations on the silicon surface under the action of pulse-periodic emission from a YAG:Nd laser // Technical Physics. The Russian Journal of Applied Physics, 46(8), p. 962-967 (2001).

21. J.M. Cole, P. Humphreys and L.G. Earwaker, A melting model for pulsed laser heating of silicon // Vacuum, 34 (10/11), p. 871-874 (1984).

22. G.E. Jellison, D.H. Lowndes, D.N. Mashburn and R.F. Wood, Time-resolved reflectivity measurements on silicon and germanium using a pulsed excimer $\mathrm{KrF}$ laser heating beam // Phys. Rev. B, 34(4), p. 2407-2415 (1986).

23. A. Feltz, Amorphe und Glasartige Anorganische Festkorper. Academie Verlag, Berlin, 1983.

24. M.H. Brodsky, R.S. Title, K. Weiser, and G.D. Pettit, Structural, optical, and electrical properties of amorphous silicon films // Phys. Rev. $B, 1(6)$, p. 2632-2641 (1970).

25. Amorphous Semiconductors. Ed. by M.H. Brodsky. Springer-Verlag, Berlin, Heidelberg, New York, 1979.

26. Amorphous Semiconductor Technologies and Devices. Ed. Y. Hamakawa. OHMSHA, Ltd., 1983. 